\title{
Wireless Powered Cooperative Relaying using NOMA with Imperfect CSI
}

\author{
Dinh-Thuan Do*, Mojtaba Vaezi ${ }^{\dagger}$, and Thanh-Luan Nguyen $\ddagger$ \\ *Wireless Communications Research Group, Faculty of Electrical and Electronics Engineering, Ton Duc \\ Thang University, Ho Chi Minh City, Vietnam \\ ${ }^{\dagger}$ Department of Electrical and Computer Engineering, Villanova University, PA 19085, USA \\ ${ }_{\ddagger}^{\ddagger}$ Department of Electrical Engineering, Bach Khoa University, Vietnam \\ Email: dodinhthuan@tdt.edu.vn,mvaezi@villanova.edu, luannguyen.cce@gmail.com
}

\begin{abstract}
The impact of imperfect channel state (CSI) information in an energy harvesting $(\mathrm{EH})$ cooperative non-orthogonal multiple access (NOMA) network, consisting of a source, two users, and an EH relay is investigated in this paper. The relay is not equipped with a fixed power source and acts as a wireless powered node to help signal transmission to the users. Closedform expressions for the outage probability of both users are derived under imperfect CSI for two different power allocation strategies namely fixed and dynamic power allocation. Monte Carlo simulations are used to numerically evaluate the effect of imperfect CSI. These results confirm the theoretical outage analysis and show that NOMA can outperform orthogonal multiple access even with imperfect CSI.

Index Terms-NOMA, imperfect CSI, SWIPT, energy harvest-
\end{abstract} ing, outage probability, Nakagami- $m$ fading.

\section{INTRODUCTION}

Effective radio access technology development plays a key role in achieving high data rate and enabling massive connectivity for next-generation wireless networks. Non-orthogonal multiple access (NOMA) is one of the key enabling technologies toward this goal $[1]-[5]$. NOMA improves the spectral efficiency and increases the number of connections and is seen as a promising candidate in $5 \mathrm{G}$ and beyond cellular networks.

Energy harvesting $(\mathrm{EH})$, on the other hand, is an important process toward green communication systems in which wasted or unimportant energy, e.g., sound and radio frequency signals, are captured and converted into electricity. By allowing wireless nodes to recharge their batteries from the radio frequency signals, rather than the traditional energy sources, EH can play an important role in powering wireless devices and has the ability to prolong the lifetime of the energy-constrained nodes. Wireless power transfer is one of the EH technologies in which green energy can be harvested using either the ambient signals or dedicated power sources, and has recently emerged as a potential technology to assist reduction of power consumption [6]-[8]. Simultaneous wireless information and power transfer (SWIPT) is a technology that enables transmission of both power and information at the same time. SWIPT can improve power consumption and spectral efficiency and also can be used for interference management. Understanding the theoretical aspects and potentials of SWIPT has attracted much research interest [9]-[11].

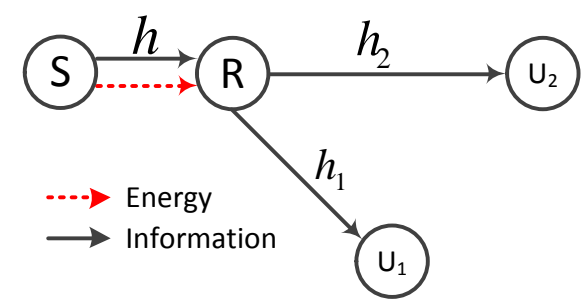

Fig. 1. A cooperative EH-NOMA consisting of a source (S) with two users $\left(\mathrm{U}_{1}\right.$ and $\mathrm{U}_{2}$ ) simultaneously through an intermediate $\mathrm{EH}$ relay $(\mathrm{R})$. Both $\mathrm{S}$ and $\mathrm{R}$ use NOMA for transmission.

Motivated by the diverse requirements of 5G systems [12], combining NOMA and SWIPT has recently been studied by different groups in various settings [13]-[17]. Specifically, the application of SWIPT to NOMA in a single-input-singleoutput system is explored in [13], in which three user-paring schemes are examined via their outage performance. The outage performance for EH-enabled NOMA relaying networks is studied in [14]. Several NOMA user selection models, including random user selection and distance-based user selections are investigated in [16], where the best-near best-far user selection scheme is promoted to enhance the outage performance. In the context of cooperative systems, SWIPT-NOMA is introduced to lengthen the lifetime of the energy-constrained relay nodes. Joint power allocation and user selection is then considered to minimize the outage probability of all users [17]. Most of the prior works have, however, assumed perfect channel state information (CSI) and Rayleigh fading channels. Due to the existence of the channel estimation errors, such an assumption is too idealistic and does not provide an insight on the performance of practical networks [18]. The analysis of NOMA networks with imperfect CSI is particularly important since the encoding and decoding of NOMA highly depends on the quality of CSI.

In this paper, we investigate the effect of imperfect CSI in an EH-NOMA network. Specifically, we consider a cooperative NOMA network, consisting of a source, two users, and an EH relay, as shown in Fig. 1, in which the relay simultaneously 
receives information and harvests energy so that it can use the harvested energy to help the source to transmit information to the users. We assume that the perfect CSI of source-relay link can be obtained whereas an imperfect CSI of the relayuser links can be obtained. We drive closed-form expressions for the outage probabilities of the NOMA users under the above assumptions and for two different power allocation schemes, namely fixed and dynamic cases. The obtained results obviously include the Rayleigh fading case by setting $m=1$. The outage probabilities of the imperfect CSI case are then compared with those of the perfect CSI to evaluate and better understand the effect of imperfect CSI.

The paper is organized as follows. We describe the system model in Section III and evaluate outage probability of the users under fixed power allocation and dynamic power allocation in Section III and Section IV, respectively. The effect of imperfect successive interference cancellation is studied in Section V. Simulation results are presented in Section $\mathrm{VI}$ and the paper is concluded in Section VII

Throughout this paper, $\mathbb{P}\{\cdot\}$ is the probability measure, and $f_{Z}(\cdot)$ and $F_{Z}(\cdot)$ denotes the probability density function (PDF) and the cumulative distribution function (CDF) of random variable $Z$, respectively.

\section{SySTEM MODEL}

Consider the cooperative NOMA network depicted in Fig. 1 . consisting of a source communicating with two users through an intermediate EH relay. The relay uses decode-and-forward relaying to assist the transmission between the source and the two users. Both the source and the relay use NOMA to transmit the users signals. All nodes are equipped with a single antenna and operate in half-duplex mode [5], [19]. We assume that perfect CSI of the source-relay link is available whereas the estimation of the relay-user links is imperfect. This is a step towards a more practical setting in which CSI at the relay is obtained via channel estimation and thus is subject to errors, unlike most of the previous works in EH-NOMA. All channels experience independent and identically distributed Rayleigh fading with zero mean and unit variance; hence, the channel coefficients $h$ and $h_{n}, n \in\{1,2\}$, are modeled as complex Gaussian random variables $\mathcal{C N}(0,1)$. 1 We also define $\tau_{1}=2^{2 R_{1}}-1, \tau_{2}=2^{2 R_{2}}-1$ and $\tau_{0}=2^{2 R_{1}+2 R_{2}}-1$, where $R_{1}$ and $R_{2}$ (bits/s/Hz) are the target data rates for user 1 and user 2 , respectively.

The transmission from the source to users consist of two phases. During the first phase, the source transmits a superimposed signal, $x_{s} \triangleq \sqrt{\xi} s_{2}+\sqrt{1-\xi} s_{1}$, to both users through the relay, where $\xi \in[0,1]$ denotes the power allocation at the source. Let us define $P \triangleq \frac{P_{s}}{\sigma^{2}}$ and $P^{\prime} \triangleq \frac{P_{r}}{\sigma^{2}}$ in which $P_{s}$ and $P_{r}$ is the transmit power of the source and the relay nodes, respectively, and $\sigma^{2}$ is the variance of the additive white Gaussian noise at the relay and users. Similar to [17],

\footnotetext{
${ }^{1}$ For the fixed power allocation in Section III the source-relay link is assumed to experience Nakagami- $m$ fading which is more general than Rayleigh fading.
}

maximum transmit power to ensure signal decoding at the relay is given as

$$
P^{\prime}=\frac{P_{r}}{\sigma^{2}}=\eta\left(P g-\tau_{0}\right), \quad g>\frac{\tau_{0}}{P}
$$

in which $\eta \in[0,1]$ denotes the energy harvesting efficiency, $g \triangleq|h|^{2} d^{-\alpha}$ where $d$ is the distance between the source and the relay, and $\alpha$ is the path loss exponent. Similar to the source, the relay also adopts NOMA for transmission.

During the second phase, the relay transmits a superposition of the decoded signals, $x_{r}=\sqrt{\delta} s_{2}+\sqrt{1-\delta} s_{1}$, to both users, where $\delta \in[0,1]$ is the power allocation coefficient at the relay.

Due to imperfect CSI, the estimated channel gains of the relay-user links are given as $\hat{h}_{n}=h_{n} d_{n}^{-\alpha / 2}+e_{n}(n=1,2)$, where $d_{n}$ is the distance between the relay and user $n$, $e_{n} \sim \mathcal{C N}\left(0, \sigma_{e}^{2}\right)$ is the channel estimation error and $\hat{h}_{n}$ is the estimated channel coefficients. We assume $e_{n}$ is independent of $h_{n}$, thus $\hat{h}_{n}$ can be modeled as a complex Gaussian random variable with zero mean and variance $d_{n}^{-\alpha}+\sigma_{e}^{2}$. Now, without loss of generality, let us order the estimated channel as $\left|\hat{h}_{1}\right|^{2}>\left|\hat{h}_{2}\right|^{2}$. Thus, the instantaneous signal-to-interferenceplus-noise-ratio (SINR) of user 2 to detect its own signal is obtained as

$$
\gamma_{2}=\frac{\delta P^{\prime} g_{2}}{(1-\delta) P^{\prime} g_{2}+P^{\prime} \sigma_{e}^{2}+1},
$$

in which $g_{n} \triangleq\left|\hat{h}_{n}\right|^{2}$. In NOMA, optimal decoding order depends on the channel gains [18]; the stronger user (user 1) first decodes the weaker user's (user 2's) signal and next its own signal after successive interference cancellation (SIC) of user 2's signal. The instantaneous SINR to detect $s_{2}$ at user 1 is given by

$$
\gamma_{1 / 2}=\frac{\delta P^{\prime} g_{1}}{(1-\delta) P^{\prime} g_{1}+\sigma_{e}^{2} P^{\prime}+1} .
$$

If $\gamma_{1 / 2} \geq \tau_{2}$, user 1 can decode $s_{2}$ and remove it from the received signal using SIC. Therefore, the instantaneous signalto-noise ratio (SNR) at user 1 to decode $s_{1}$ after $\mathrm{SIC}^{2}$ is given by

$$
\gamma_{1}=\frac{(1-\delta) P^{\prime} g_{1}}{P^{\prime} \sigma_{e}^{2}+1}
$$

\section{FiXed Power Allocation}

In this section, we consider a fixed power allocation scenario for both users. Since the source node may have line-of-sight with the relay, the source-relay link is assumed to experience Nakagami- $m$ fading, a more general case of Rayleigh fading in which the shape factor is $m=1$. Consequently, the outage probability at user $1, p_{\mathrm{F}}^{(1)}$ is expressed as

$$
p_{\mathrm{F}}^{(1)}=1-\mathbb{P}\left\{g>\frac{\tau_{0}}{P}, \gamma_{1 / 2}>\tau_{2}, \gamma_{1}>\tau_{1}\right\} .
$$

\footnotetext{
${ }^{2}$ Due to imperfect CSI, there will be another error term in the denominator of (3), i.e., SIC cannot be perfect. Equivalently, this can be seen as a reduction in the target rate $\tau_{1}$. This will be discussed in Section $\mathrm{V}$
} 
Lemma 1. For the FPA case, the outage probability of user 1 when $\delta>\frac{\tau_{2}}{\tau_{2}+1}$ can be expressed as

$$
\begin{aligned}
p_{\mathrm{F}}^{(1)}=1 & -\sum_{i=0}^{m-1}\left(\begin{array}{c}
m-1 \\
i
\end{array}\right) e^{-\frac{\tau_{0} m}{d^{-\alpha} P}}\left(\frac{\tau_{0} m}{d^{-\alpha} P}\right)^{m-i-1} \\
& \times \sum_{k=1}^{3} \frac{(-1)^{k-1}}{2^{i} \Gamma(m)} e^{-\frac{\Phi_{n 1}}{\Omega_{k}}} \beta_{n, k}^{i+1} K_{i+1}\left(\beta_{n, k}\right),
\end{aligned}
$$

in which $K_{v}(z)$ is the $v^{\text {th }}$ order modified Bessel function of the second kind [20], $\beta_{n, k}=2 \sqrt{\frac{m}{d^{-\alpha} P} \frac{\Phi_{\mathrm{n} 2}}{\Omega_{k}}}$ in which

$$
\left[\begin{array}{ll}
\Phi_{11} & \Phi_{12} \\
\Phi_{21} & \Phi_{22}
\end{array}\right] \triangleq\left[\begin{array}{cc}
\frac{\tau_{2}}{\delta\left(\tau_{2}+1\right)-\tau_{2}} & \frac{\tau_{1}}{1-\delta}
\end{array}\right]^{T}\left[\begin{array}{ll}
\sigma_{e}^{2} & \frac{1}{\eta}
\end{array}\right],
$$

$\Omega_{1}=d_{2}^{-\alpha}+\sigma_{e}^{2}, \Omega_{2}=\frac{\Omega_{1} \Omega_{3}}{\Omega_{1}+\Omega_{3}}$ and $\Omega_{3}=d_{1}^{-\alpha}+\sigma_{e}^{2}$. Also, note that $p_{F}^{(1)}=1$ when $\delta \leq \frac{\tau_{2}}{\tau_{2}+1}$.

Proof. See Appendix A.

Similar to 5 , we have $p_{\mathrm{F}}^{(2)}=1-\mathbb{P}\left\{P^{\prime}>0, \gamma_{2}>\tau_{2}\right\}$ as the outage probability for user 2 . For $\delta>\frac{\tau_{2}}{\tau_{2}+1}, p_{\mathrm{F}}^{(2)}$ can be expressed as

$$
\begin{aligned}
p_{\mathrm{F}}^{(2)}=1-\sum_{i=0}^{m-1} & \left(\begin{array}{c}
m-1 \\
i
\end{array}\right) e^{-\frac{\tau_{0} m}{d^{-\alpha} P}}\left(\frac{\tau_{0} m}{d^{-\alpha} P}\right)^{m-i-1} \\
& \times \frac{1}{2^{i} \Gamma(m)} e^{-\frac{\Phi_{11}}{\Omega_{2}}} \beta_{1,2}^{i+1} K_{i+1}\left(\beta_{1,2}\right) .
\end{aligned}
$$

Note that $p_{\mathrm{F}}^{(1)}=p_{\mathrm{F}}^{(2)}=1$ when $\delta \leq \frac{\tau_{2}}{\tau_{2}+1}$.

Remark 1. When the source-relay link experiences a Rayleigh fading, we can substitute $m=1$ into (6) and 8 to achieve the outage probability for both users.

Remark 2. Diversity order is zero at both users; i.e.,

$$
D_{\mathrm{F}}^{(n)}=-\lim _{P \rightarrow \infty} \frac{\log p_{\mathrm{F}}^{(n)}}{\log P}=0, \quad n=1,2 .
$$

This is because in the high SNR regime, i.e., when $P \rightarrow \infty$, we have $\beta_{n, k}^{i+1} K_{i+1}\left(\beta_{n, k}\right) \rightarrow 2^{i} \Gamma(i+1)[20]$ and $e^{-\frac{\tau_{0} m}{d^{-\alpha} P}} \rightarrow 1$. Then, $p_{\mathrm{F}}^{(1)}=1-\sum_{k=1}^{3}(-1)^{k-1} e^{-\frac{\Phi_{\mathrm{n} 1}}{\Omega_{k}}}, p_{\mathrm{F}}^{(2)}=1-e^{-\frac{\Phi_{11}}{\Omega_{2}}}$, and (9) holds.

\section{DYNAMic Power Allocation}

In this section, the power allocation is adjusted at the relay to ensure signal decoding at user 2 . Since it is not tractable to adopt Nakagami- $m$ fading model on the source-relay link due to its mathematical complexity, we use the Rayleigh model in this section. By solving $\gamma_{2}=\tau_{2}$ to find $\delta$ and, noting that $1-\delta$ must be non-negative, we obtain

$$
\delta=1-\max \left\{0, \frac{P^{\prime} g_{2}-\tau_{2}\left(\sigma_{e}^{2} P^{\prime}+1\right)}{\left(1+\tau_{2}\right) P^{\prime} g_{2}}\right\} .
$$

It should be highlighted that the performance of user 2, and consequently user fairness can be further enhanced by letting $\delta$ larger than what is given in 100 . This, however, significantly reduces the performance of user 1 and also the sum rate [18].
Based on (5) and (10), the outage probability at user 1 for the DPA case is defined as

$$
p_{\mathrm{D}}^{(1)}=1-\mathbb{P}\left\{g>\frac{\tau_{0}}{P}, g_{2}>\tau_{2}\left(\sigma_{e}^{2}+\frac{1}{P^{\prime}}\right), \gamma_{1}>\tau_{1}\right\} .
$$

Lemma 2. For the DPA case, the outage probability of user 1 is given by

$$
p_{\mathrm{D}}^{(1)}=1-e^{-\sigma_{e}^{2} \frac{\tau_{0}}{\Omega_{2}}-\frac{\tau_{0} d^{\alpha}}{P}} \omega_{0} K_{1}\left(\omega_{0}\right)-\Upsilon_{\mathrm{II}},
$$

in which $\Upsilon_{\mathrm{II}}$ is defined in $B-3$ and $\omega_{\ell}=2 \sqrt{\frac{1}{\eta P d^{-\alpha}} \frac{\tau_{\ell}}{\Omega_{2}}}, \ell \in$ $\{0,2\}$.

\section{Proof. See Appendix B.}

Further, the outage probability at user 2 for the DPA case is given as

$$
p_{\mathrm{D}}^{(2)}=1-\mathbb{P}\left\{g>\frac{\tau_{0}}{P}, g_{2}>\tau_{2}\left(\sigma_{e}^{2}+\frac{1}{P^{\prime}}\right)\right\} .
$$

Then it is straightforward to show that the outage probability of user 2 is obtained by

$$
p_{\mathrm{D}}^{(2)}=1-e^{-\sigma_{e}^{2} \frac{\tau_{2}}{\Omega_{2}}-\frac{d^{\alpha} \tau_{0}}{P}} \omega_{2} K_{1}\left(\omega_{2}\right) .
$$

Remark 3. Similar to the FPA case in Remark 1, the diversity order is zero in the DPA case too.

\section{THE EFFECT OF IMPERFECT SIC}

Obtaining (4) in Section II] we have assumed that SIC is perfect. However, since the CSI of the relay-to-user channels is imperfect, SIC cannot be error-free in practice. In this section, we investigate the detrimental effect of imperfect CSI in SIC and outage probabilities. It should be highlighted that imperfect SIC can significantly affect the performance of the system even if the channel estimation is accurate.

To study the joint impact of imperfect SIC and imperfect CSI, an analysis similar to that of [21] can be carried out here. However, applying this analysis for the DPA-NOMA scheme is not straightforward and requires large modifications which cannot be included due to the space limit. Recall that in the DPA-NOMA case, the relay does not have the knowledge of the SIC process at user 1 and thus can only adjust the power allocation in a perfect SIC manner. Thus, we only discuss the impact of imperfect SIC to the FPA-NOMA scheme.

We know that the received signal at user 1 (i.e. the stronger user) is given by

$$
y_{1}=\sqrt{P_{r}}\left(\sqrt{\delta} s_{2}+\sqrt{1-\delta} s_{1}\right)\left(\hat{h}_{1}+e_{1}\right)+n_{1} .
$$

Then, the received signal after the SIC process is given by

$$
\begin{aligned}
\hat{y}_{1}= & \sqrt{P_{r}(1-\delta)} s_{1} \hat{h}_{1}+\sqrt{P_{r} \delta} \hat{h}_{1}\left(s_{2}-\hat{s}_{2}\right) \\
& +\left(\sqrt{P_{r}(1-\delta)} s_{1}+\sqrt{P_{r} \delta} s_{2}\right) e_{1}+n_{1},
\end{aligned}
$$

where $\hat{s}_{2}$ is the estimated signal of the user 2 at user 1 . Let $\sigma_{i c}^{2} \triangleq \mathbb{E}\left[\left|s_{2}-\hat{s}_{2}\right|^{2}\right]$ denote the expected residual power level after SIC, $\sigma_{i c}^{2}=0$ would refer to perfect SIC case in which $s_{2}=\hat{s}_{2}$. 

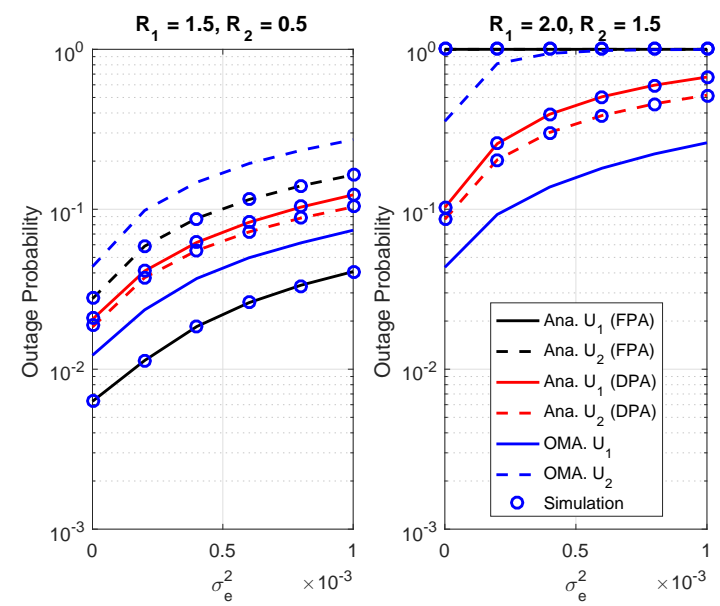

Fig. 2. Outage probability of FPA-NOMA/DPA-NOMA cases versus $\sigma_{e}^{2}$, where $P_{s}=15 \mathrm{~dB}$.

The SINR at user 1 after imperfect SIC, i.e., the modified version of (3), becomes

$$
\gamma_{1}=\frac{(1-\delta) P^{\prime} g_{1}}{P^{\prime} \sigma_{e}^{2}+P^{\prime} \delta g_{1} \sigma_{i c}^{2}+1} .
$$

Then, for the FPA-NOMA with imperfect SIC, Lemma 1 requires a slight modification. Specifically, (7) should be replace by

$\left[\begin{array}{ll}\Phi_{11} & \Phi_{12} \\ \Phi_{21} & \Phi_{22}\end{array}\right] \triangleq\left[\frac{\tau_{2}}{\delta\left(\tau_{2}+1\right)-\tau_{2}}, \frac{\tau_{1}}{1-\delta\left(\tau_{1} \sigma_{i c}^{2}+1\right)}\right]^{T}\left[\sigma_{e}^{2}, \frac{1}{\eta}\right]$

Note that $p_{\mathrm{F}}^{(1)}=p_{\mathrm{F}}^{(2)}=0$ when either $\delta \leq \frac{\tau_{2}}{\tau_{2}+1}$ or $\delta \geq \frac{1}{\tau_{1} \sigma_{i c}^{2}+1}$.

Proof. Taking similar steps as in Appendix A, we can get the exact outage probability for this case. Specifically, $p_{\mathrm{F}}^{(1)}$ and $p_{\mathrm{F}}^{(2)}$ are simply derived by substitution into (A-2) which gives

$$
\begin{aligned}
\Lambda_{\max } & = \\
& \begin{cases}\Phi_{11}+\frac{\Phi_{12}}{P g-\tau_{0}}, & \frac{\tau_{2}}{\tau_{2}+1}<\delta<\min \left[\frac{\tau_{0}-\tau_{1}}{\tau_{0}+\tau_{1} \tau_{2} \sigma_{i c}^{2}}, \frac{1}{\tau_{1} \sigma_{i c}^{2}+1}\right] \\
\Phi_{21}+\frac{\Phi_{22}}{P g-\tau_{0}}, & \frac{1}{\tau_{1} \sigma_{i c}^{2}+1}>\delta \geq \frac{\tau_{0}-\tau_{1}}{\tau_{0}+\tau_{1} \tau_{2} \sigma_{i c}^{2}}\end{cases}
\end{aligned}
$$

\section{NUMERICAL RESULTS}

In this section, numerical results are presented to validate the analytic results and to evaluate the outage performance of the downlink EH-NOMA network. The source-relay and relayusers links are considered under Rayleigh fading environment, $\sigma^{2}=-30 \mathrm{~dB}$ and $\delta=0.8$. The distances between the Relay

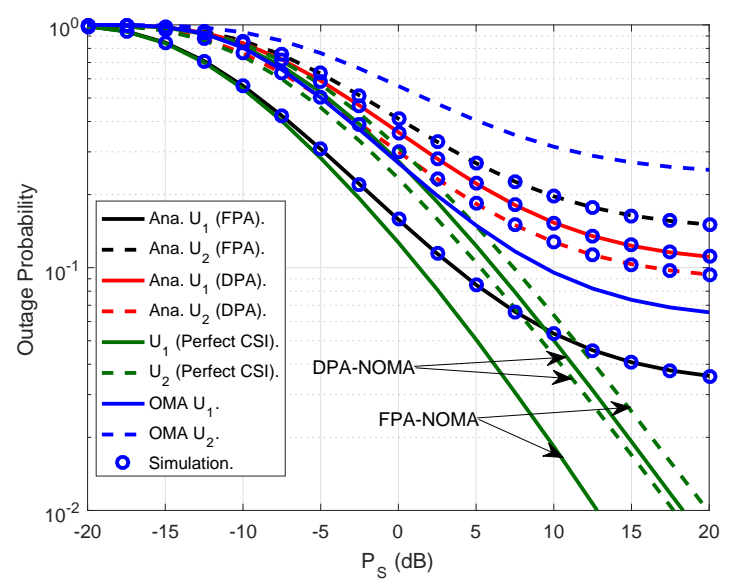

Fig. 3. Outage probability of FPA-NOMA/DPA-NOMA case versus $P_{s}$, where $R_{2}=0.5, R_{1}=1.5$ and $\sigma_{e}^{2}=0.001$.

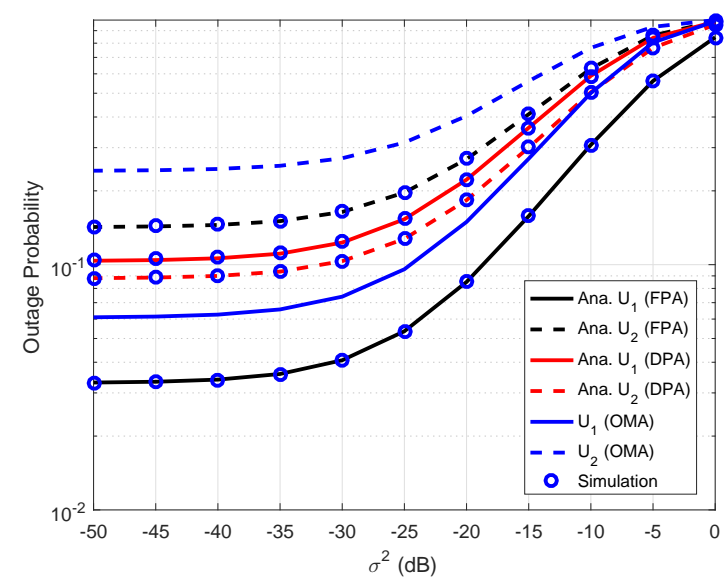

Fig. 4. Outage probability of FPA-NOMA/DPA-NOMA for imperfect and perfect CSI cases versus noise power, where $R_{2}=0.5, R_{1}=1.5, \sigma_{e}^{2}=$ 0.001 and $P_{s}=15 \mathrm{~dB}$.

to each node are $d=1, d_{1}=1, d_{2}=10$ and the path loss exponent is $\alpha=2$. Note that the target data rates from Fig. 2 to Fig. 4 i.e. $R_{1}$ and $R_{2}$, are given in terms of bits/s/Hz.

As can be seen from Fig. 2 to Fig. 4, the analytical curves match the simulation curves. In Fig. 2, the outage probability at user 1 and user 2 increases as $R_{1}$ and $R_{2}$ increase. Choosing large values for $R_{1}$ and $R_{2}$ could make the outage event always occur, i.e. the outage probabilities become one. Figures 3 and 4 confirm that as $P_{s}$ increases the outage probability decreases. Specifically, in Fig. 3 . FPANOMA results in a higher outage at the weak user but provides much higher success probability for the strong user than DPANOMA. Unlike that in Fig. 4, it is seen that FPA-NOMA can provide better performance for both users than OMA. Further, it is observed that an outage floor appears in the outage probability due to the channel estimation errors.

Further, the impact of channel estimation error $\sigma_{e}^{2}$ and SIC 


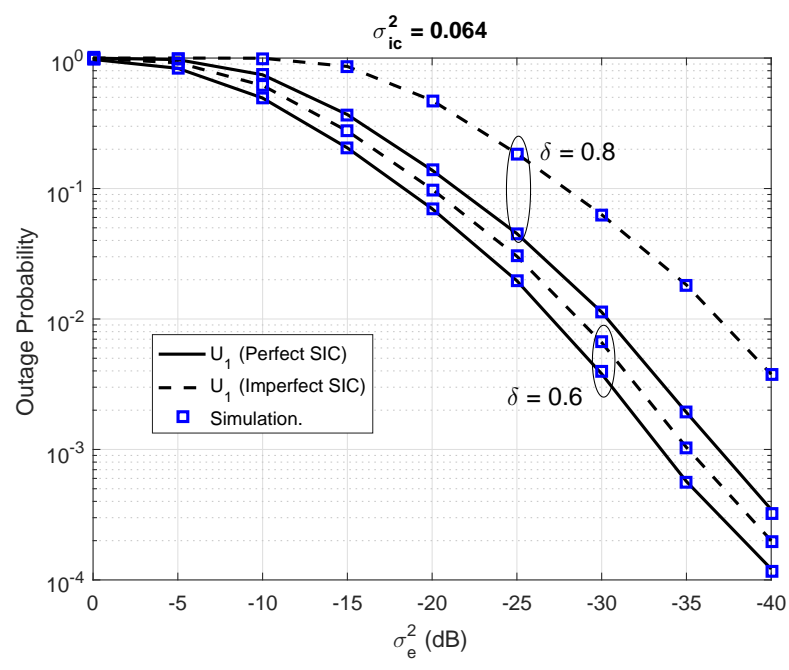

Fig. 5. Outage probability of FPA-NOMA case versus $\sigma_{e}^{2}$, where $R_{2}=$ $0.5, R_{1}=1.5, d=d_{1}=1, d_{2}=10$ and $P_{s}=30 \mathrm{~dB}$

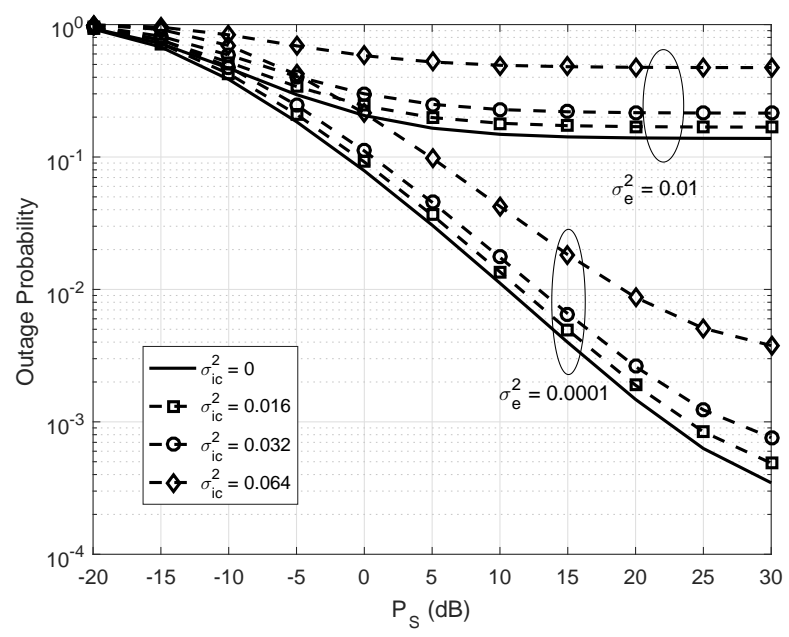

Fig. 6. Outage probability of FPA-NOMA case versus $P_{s}$, where $R_{2}=$ $0.5, R_{1}=1.5, d=d_{1}=1$ and $d_{2}=10$.

error $\sigma_{i c}^{2}$ is investigated in Fig. 5 and Fig. 6. It is observed that a higher channel estimation accuracy leads to a higher degradation in the outage performance with the same value of $\sigma_{i c}^{2}$. For example, for $\sigma_{e}^{2}=-40 \mathrm{~dB}$ the outage probability with imperfect SIC is reduced more than 10 times from that with perfect SIC, but for $\sigma_{e}^{2}=-20 \mathrm{~dB}$ this reduction is about 4 times. However, one can improve the system performance as well as the gap between the perfect and imperfect SIC by increasing the power allocated for user 1 (decreasing $\delta$ ). In fact, as $\delta$ decreases (but $\delta>\frac{\tau_{2}}{\tau_{2}+1}$ holds), the effect of imperfect SIC become less significant, e.g., resulting only 1.7 times reduction in the performance at $\sigma_{e}^{2}=-40$ for $\delta=0.6$. Further, by increasing the transmit power, the outage performance can be improved but reaches different outage floor depending on $\sigma_{i c}^{2}$ as depicted in Fig. 6

\section{CONCLUSION}

We have examined the outage performance of EH-NOMA networks with imperfect CSI and imperfect SIC. We have derived closed-form expressions for the outage probability at each user. Simulation results confirm the analytic results and show that NOMA can improve the outage probability of both users. In addition, it is shown that FPA-NOMA achieves better performance for the stronger user than DPA-NOMA but poor fairness. Further, DPA-NOMA provides a higher success probability for the weaker than FPA-NOMA and a better fairness. It is also confirmed that FPA-NOMA can outperform orthogonal multiple access schemes.

\section{APPENDIX A}

From (5) the outage probability of user 1 can be written as

$$
p_{\mathrm{F}}^{(1)}=1-\mathbb{P}\left\{g>\frac{\tau_{0}}{P}, g_{1}>\Lambda_{\max }\right\},
$$

where

$$
\begin{aligned}
\Lambda_{\max } & =\max \left(\Phi_{11}+\frac{\Phi_{12}}{P g-\tau_{0}}, \Phi_{21}+\frac{\Phi_{22}}{P g-\tau_{0}}\right) \\
& =\left\{\begin{array}{lc}
\Phi_{11}+\frac{\Phi_{12}}{P g-\tau_{0}}, & \frac{\tau_{2}}{\tau_{2}+1}<\delta<\frac{\tau_{0}-\tau_{1}}{\tau_{0}} \\
\Phi_{21}+\frac{\Phi_{22}}{P g-\tau_{0}}, & \delta \geq \frac{\tau_{0}-\tau_{1}}{\tau_{0}}
\end{array}\right.
\end{aligned}
$$

where the last step can be verified in view of (7). Since $F_{g_{1}}(\gamma)=1-\sum_{k=1}^{3}(-1)^{k-1} \exp \left(-\frac{\gamma}{\Omega_{k}}\right)$ is the CDF of $g_{1}$ and $f_{g}(\gamma)=\frac{m^{m} d^{\alpha m}}{\Gamma(m)} \gamma^{m-1} \exp \left(-m d^{\alpha} \gamma\right)$ is the PDF of $g$ [17], $p_{\mathrm{F}}^{(1)}$ can be expressed as

$$
\begin{aligned}
p_{\mathrm{F}}^{(1)}=1 & -\sum_{k=1}^{3}(-1)^{k-1} \frac{m^{m}}{\Gamma(m) d^{-\alpha m}} \\
& \times \underbrace{\int_{\tau_{0} / P}^{\infty} z^{m-1} \exp \left(-\frac{\Lambda_{\max }}{\Omega_{k}}-\frac{m z}{d^{-\alpha}}\right) d z}_{\mathbf{X}_{m}} .
\end{aligned}
$$

Next, using $\mathrm{A}-2$ for $\frac{\tau_{2}}{\tau_{2}+1}<\delta<\frac{\tau_{0}-\tau_{1}}{\tau_{0}}$, with the help of [20. Eq. 3.471.9], the integral in $\mathrm{A}-3$ is evaluated as

$$
\begin{aligned}
\mathrm{X}_{m} & =\sum_{i=0}^{m-1}\left(\begin{array}{c}
m-1 \\
i
\end{array}\right) e^{-\frac{m \tau_{0}}{P d^{-\alpha}}}\left(\frac{\tau_{0}}{P}\right)^{m-i-1} \\
& \times 2 e^{-\frac{\Phi_{11}}{\Omega_{k}}}\left(\sqrt{\frac{\Phi_{12}}{\Omega_{k}} \frac{d^{-\alpha}}{m P}}\right)^{i+1} K_{i+1}\left(\beta_{1, k}\right),
\end{aligned}
$$

in which $\left(\begin{array}{l}n \\ k\end{array}\right)$ shows the binomial coefficient. Then, it can be checked that for $\delta \geq \frac{\tau_{0}-\tau_{1}}{\tau_{0}}$, the value of $\mathrm{X}_{m}$ is obtained by replacing $\Phi_{11}$ and $\Phi_{12}$ with $\Phi_{21}$ and $\Phi_{22}$ in A-4, respectively. Substituting (A-4) into (A-3), respecting the value of $\delta$, after some mathematical manipulations the proof is completed. 


$$
\begin{aligned}
\Upsilon_{\mathrm{II}} & =\sum_{i \in\{1,3\}} \frac{d^{\alpha}}{\Omega_{\bar{i}} \eta P} e^{-\frac{\tau_{0}}{P d^{-\alpha}}} \int_{0}^{\tau_{1}+\tau_{1} \tau_{2}} e^{-\mathrm{H}_{\bar{i}}(\rho) \sigma_{e}^{2}} \int_{0}^{\infty}\left(\sigma_{e}^{2}+\frac{1}{v}\right) \exp \left(-\mathrm{H}_{\bar{i}}(\rho)-\frac{v}{\eta P d^{-\alpha}}\right) d v d \rho \\
& \stackrel{(a)}{=} \sum_{i \in\{1,3\}} \frac{1}{\Omega_{\bar{i}}} e^{-\frac{\tau_{0}}{P d^{-\alpha}}} \int_{0}^{\tau_{1}+\tau_{1} \tau_{2}} \underbrace{e^{-\mathrm{H}_{\bar{i}}(\rho) \sigma_{e}^{2}}\left[\sigma_{e}^{2} 2 \sqrt{\frac{\mathrm{H}_{\bar{i}}(\rho)}{\eta P d^{-\alpha}}} K_{1}\left(2 \sqrt{\frac{\mathrm{H}_{\bar{i}}(\rho)}{\eta P d^{-\alpha}}}\right)+\frac{2}{\eta P d^{-\alpha}} K_{0}\left(2 \sqrt{\frac{\mathrm{H}_{\bar{i}}(\rho)}{\eta P d^{-\alpha}}}\right)\right]}_{\triangleq h_{i}(\rho)} d \rho \\
& \stackrel{(b)}{\approx} \sum_{i \in\{1,3\}} \frac{1}{\Omega_{\bar{i}}} e^{-\frac{\tau_{0}}{P d^{-\alpha}}} \frac{\tau_{1}+\tau_{1} \tau_{2}}{2} \sum_{j=1}^{J} \frac{\pi}{j}\left|\sin \left(\frac{2 j-1}{2 J} \pi\right)\right| h_{i}\left(\rho_{n}\right),
\end{aligned}
$$

where $\rho_{n}=\frac{\tau_{1}+\tau_{1} \tau_{2}}{2}\left[1+\cos \left(\frac{2 j-1}{2 J} \pi\right)\right]$ and $J$ is the trade-off coefficient reflecting the approximation accuracy.

\section{APPENDIX B}

From (10) the outage probability of user 1 in (11) can be further expressed as

$$
\begin{aligned}
p_{\mathrm{F}}^{(1)}= & 1-\mathbb{P}\left\{g_{1}>g_{2}>\tau_{0}\left(\frac{1}{P^{\prime}}+\sigma_{e}^{2}\right), g>\frac{\tau_{0}}{P}\right\} \\
& -\mathbb{P}\left\{g_{1}>\frac{\tau_{1}\left(P^{\prime} \sigma_{e}^{2}+1\right)\left(1+\tau_{2}\right) g_{2}}{P^{\prime} g_{2}-\left(P^{\prime} \sigma_{e}^{2}+1\right) \tau_{2}}, g>\frac{\tau_{0}}{P},\right. \\
& \left.\tau_{2}\left(\frac{1}{P^{\prime}}+\sigma_{e}^{2}\right)<g_{2}<\tau_{0}\left(\frac{1}{P^{\prime}}+\sigma_{e}^{2}\right)\right\} .
\end{aligned}
$$

Note that $f_{g_{2}, g_{1}}(x, y)=\sum_{i \in\{1,3\}} \frac{1}{\Omega_{\bar{i}} \Omega_{i}} \exp \left(-\frac{x}{\Omega_{\bar{i}}}-\frac{y}{\Omega_{i}}\right)$ and $\tau_{1}+\tau_{2}+\tau_{1} \tau_{2}=\tau_{0}$. The proof of the first probability is not shown in this paper as it involves simpler mathematical computations than the second one and can be easily derived by adopting steps in Appendix A. The second probability in (B-1), denoted as $\Upsilon_{\mathrm{II}}$, is obtained as follows

$$
\Upsilon_{\mathrm{II}}=\int_{\frac{\tau_{0}}{P}}^{\infty} \int_{\tau_{2}\left(\sigma_{e}^{2}+\frac{1}{\eta\left(P z-\tau_{0}\right)}\right)}^{\tau_{0}\left(\sigma_{e}^{2}+\frac{1}{\eta\left(P z-\tau_{0}\right)}\right)} \int_{\nu(x, z)}^{\infty} f_{g_{2}, g_{1}}(x, y) f_{g}(z) d x d y d z,
$$

where $\nu(x, z) \triangleq \frac{\tau_{1}\left(1+\tau_{2}\right)\left(\eta\left(P z-\tau_{1}\right) \sigma_{e}^{2}+1\right) x}{\eta\left(P z-\tau_{0}\right) x-\tau_{2}\left(\eta\left(P z-\tau_{0}\right) \sigma_{e}^{2}+1\right)}$

By letting $v=\eta\left(P z-\tau_{0}\right)$ and then $\rho=\frac{v}{v \sigma_{e}^{2}+1} x-\tau_{2}$, the above equality can be expressed by (B.3), where $\mathrm{H}_{i}(\rho) \triangleq$ $\left(\frac{\tau_{1}\left(1+\tau_{2}\right)}{\rho} \frac{1}{\Omega_{\bar{i}}}+\frac{1}{\Omega_{i}}\right)\left(\rho+\tau_{2}\right), \bar{i}=3 / i$, (a) is due to 20 Eq.3.471.9] and (b) is obtained by using Gaussian-Chebyshev quadrature [17].

\section{REFERENCES}

[1] M. Vaezi, Z. Ding, and H. V. Poor, Multiple Access Techniques for $5 G$ Wireless Networks and Beyond. Springer, 2019.

[2] Y. Saito, Y. Kishiyama, A. Benjebbour, T. Nakamura, A. Li, and K. Higuchi, "Non-orthogonal multiple access (NOMA) for cellular future radio access," in Proc. IEEE 77th VTC Spring, pp. 1-5, 2013.

[3] Z. Ding, Z. Yang, P. Fan, and H. V. Poor, "On the performance of non-orthogonal multiple access in $5 \mathrm{G}$ systems with randomly deployed users," IEEE Signal Proc. Lett., vol. 21, no. 12, pp. 1501-1505, 2014.

[4] W. Shin, M. Vaezi, B. Lee, D. J. Love, J. Lee, and H. V. Poor, "Nonorthogonal multiple access in multi-cell networks: Theory, performance, and practical challenges," IEEE Commun. Mag., vol. 55, no. 10, pp. 176 183,2017

[5] X. Yue, Y. Liu, S. Kang, A. Nallanathan, and Z. Ding, "Exploiting full/half-duplex user relaying in noma systems," IEEE Trans. Commun., vol. 66 , no. 2 , pp. 560-575, 2018.
[6] M. Piñuela, P. D. Mitcheson, and S. Lucyszyn, "Ambient RF energy harvesting in urban and semiurban environments," IEEE Trans. Microw. Theory Techn., vol. 61, pp. 2715-2726, 2013.

[7] C. Valenta and G. Durgin, "Harvesting wireless power: Survey of energy-harvester conversion efficiency in far-field, wireless power transfer systems," IEEE Microw. Mag., vol. 15, pp. 108-120, 2014.

[8] S. Bi, C. K. Ho, and R. Zhang, "Wireless powered communication: opportunities and challenges," IEEE Commun. Mag., vol. 53, pp. 117$125,2015$.

[9] R. Zhang and C. K. Ho, "MIMO broadcasting for simultaneous wireless information and power transfer," IEEE Trans. Wireless Commun., vol. 12, pp. 1989-2001, 2013.

[10] L. Liu, R. Zhang, and K.-C. Chua, "Wireless information transfer with opportunistic energy harvesting," IEEE Trans. Wireless Commun., vol. 12, pp. 288-300, 2013.

[11] J. Park and B. Clerckx, "Joint wireless information and energy transfer in a two-user MIMO interference channel," IEEE Trans. Wireless Commun., vol. 12, pp. 4210-4221, 2013.

[12] ITU, "Minimum requirements related to technical performance for IMT2020 radio interface(s)." https://www.itu.int/md/R15-SG05-C-0040/en 2017. [Online; accessed August 2008].

[13] Y. Liu, Z. Ding, M. Elkashlan, and H. V. Poor, "Cooperative nonorthogonal multiple access with simultaneous wireless information and power transfer," IEEE J. Sel. Areas Commun., vol. 34, no. 4, pp. 938953, 2016.

[14] W. Han, J. Ge, and J. Men, "Performance analysis for NOMA energy harvesting relaying networks with transmit antenna selection and maximal-ratio combining over Nakagami-m fading," IET Commun., vol. 10 , no. 18 , pp. $2687-2693,2016$.

[15] X.-X. Nguyen and D.-T. Do, "Maximum harvested energy policy in fullduplex relaying networks with SWIPT," Int. J. Commun. Sys., no. 7, pp. 1-16, 2017.

[16] N. T. Do, D. B. Da Costa, T. Q. Duong, and B. An, "A BNBF user selection scheme for NOMA-based cooperative relaying systems with SWIPT," IEEE Commun. Lett., vol. 21, no. 3, pp. 664-667, 2017.

[17] Z. Yang, Z. Ding, P. Fan, and N. Al-Dhahir, "The impact of power allocation on cooperative non-orthogonal multiple access networks with SWIPT," IEEE Trans. Wireless Commun., vol. 16, no. 7, pp. 4332-4343, 2017.

[18] M. Vaezi, R. Schober, Z. Ding, and H. V. Poor, "Non-orthogonal multiple access: Common myths and critical questions," https://arxiv.org/abs/ 1809.07224

[19] T. N. Do, D. B. da Costa, T. Q. Duong, and B. An, "Improving the performance of cell-edge users in noma systems using cooperative relaying," IEEE Trans. Commun., vol. 66, no. 5, pp. 1883-1901, 2018.

[20] A. Jeffrey and D. Zwillinger, Table of integrals, series, and products. Academic Press, 2007.

[21] X. Chen, Z. Zhang, C. Zhong, R. Jia, and D. W. K. Ng, "Fully nonorthogonal communication for massive access," IEEE Trans. Commun., vol. 66, no. 4, pp. 1717-1731, 2018. 